\title{
Time-Dependent Reliability of Concrete Structure in Chloride Environment
}

\author{
JIANG Qiong Ming ${ }^{1, a}$, SHI Kai, ${ }^{2, b}$ and LAN Sheng Ning ${ }^{1, c}$ \\ ${ }^{1}$ College of Civil Engineering and Architecture, Qinzhou University, Qinzhou, 535011, China \\ ${ }^{2}$ China Nuclear Industry 24 Construction CO., LTD. Shanghai, 201803, China \\ ajiangqiongming@qzhu.edu.cn, b156571933@qq.com, c lan2530@163.com
}

\begin{abstract}
Keywords: chloride; concrete; reliability; age factor
Abstract. In this paper, the age factor of chloride diffusion coefficient of concrete is considered, as well as the randomness of the chloride diffusion coefficient, concrete cover depth, surface concentration, threshold concentration and age factor. Based on the First Order Reliability Method, a time-dependent reliability analysis model for concrete structures under chloride conditions is established. The results show that the time-dependent reliability model is in good agreement with the Monte Carlo sampling method. The results of example show that the corner region of the concrete structure must be designed and calculated based on the two-dimensional diffusion model, and the attenuation characteristics and randomness must be considered for the concrete structures in chloride environment.
\end{abstract}

\section{Introduction}

The passive film around steel bar will be destroyed when the chloride concentration at the surface of the steel bar reaches the critical value. In fact, the service life of concrete structures cannot be accurately predicted. This process is affected by a large number of factors, and the relationship between them is not very clear [1]. Therefore, considering the randomness of the parameters during the diffusion process of the chloride in concrete structure and adopting reliability analysis of the durability of the structure is closer to the actual situation. The randomness of parameters mainly comes from the concrete materials, external environment and construction process of concrete structure materials. The randomness of these parameters will lead to the lack of design and maintenance of the concrete structure, which in turn will seriously shorten the service life and increase the whole life cycle costs [4]. In the existing research, the main random parameters are four parameters, the cover depth, the chloride diffusion coefficient, the surface concentration and the threshold concentration [2]. In addition, there are also factors such as initial chloride concentration and age factor of chloride diffusion in concrete, and other factors [3].

In [4], the reliability of the concrete structure was analyzed using the first order reliability method. Through the importance coefficient analysis, the influence of the randomness of each parameter on the durability of the structure was compared. Some of the literature only analyzed the one-dimensional chloride diffusion [15], and some did not take into account the effects of the denaturation of the parameters [4]. At the level of structure, many concrete structures are eroded by the chloride in two or even multiple directions for the actual concrete structures [14], such as beam-column members [6]-[9] and cracked concrete structures [10]-[11]. The results of experimental tests [12] and numerical model studies [13] have shown that the chloride concentration in two-dimensional diffusion regions is higher than that in the one-dimensional diffusion regions.

This paper considers the age factor of chloride diffusion coefficient of concrete, and simultaneously considers the randomness of the chloride diffusion coefficient, cover depth, surface concentration, threshold concentration, and age factor. The reliability method was used to analyze the time-dependent reliability of the concrete structure under the two-dimensional diffusion, and sensitivity coefficients were used to compare the degree of influence of random parameters on reliability. 


\section{Analytical Model of Chloride Diffusion in Concrete}

The analytical solutions for the one-dimensional and two-dimensional diffusion equations respectively are [15]:

$$
\begin{aligned}
& C=C_{0}+\left(C_{s}-C_{0}\right)\left[1-\operatorname{erf}\left(\frac{x}{2 \sqrt{D_{a} t}}\right)\right] \\
& C=C_{0}+\left(C_{s}-C_{0}\right)\left[1-\operatorname{erf}\left(\frac{x}{2 \sqrt{D_{a} t}}\right) \operatorname{erf}\left(\frac{y}{2 \sqrt{D_{a} t}}\right)\right] \\
& D_{a}=\frac{D_{0}}{1-n}\left(\frac{t_{0}}{t}\right)^{n}
\end{aligned}
$$

Where, $C$ denotes the chloride concentration in concrete ( $\%$, the percentage of chloride in the mass of concrete is used as the unit of chloride concentration in this paper); $C_{\text {s }}$ denotes the chloride concentration on the concrete surface; $C_{0}$ denotes the initial chloride concentration in the concrete; $D_{\mathrm{a}}$ denotes the apparent diffusion coefficient $\left(\mathrm{mm}^{2} / y e a r\right) ; D_{0}$ denotes the chloride diffusion coefficient $\left(\mathrm{mm}^{2} /\right.$ year) of the concrete at $t_{0} ; n$ denotes the age factor of the diffusion coefficient; $t$ denotes the time (year) when the concrete is exposed to the chloride environment.

The apparent diffusion coefficient is an important parameter to characterize chloride transport in concrete. Due to the continuous hydration of cementitious materials in concrete and its combination with chloride ions, the pore structure of concrete is continuously dense, so the time-dependent law of the apparent diffusion coefficient is related to the exposure time of concrete in the chloride environment, and the apparent diffusion coefficient decreases with the exposure time. The value of age factor $n$ denotes the decreasing rate of apparent diffusion coefficient with exposure time. It will have $D_{a}=D_{0}$ when $n=0$, which means that the diffusion coefficient of concrete is a constant in whole life without attenuation.

\section{Reliability of Concrete Structures}

The process of chloride transports into the concrete structures in the chloride environment is random, the distribution of chloride concentration in the concrete structure is also random, leading to the deterministic method being unable to accurately solve the service life of the concrete structure. And need to use reliability analysis method. When the chloride concentration on the surface of the steel bar reaches the critical value, the passive film on the surface of the steel bar is considered to be damaged, and it is used as the starting time of corrosion of the steel bar. According to the threshold concentration $C_{\mathrm{cr}}$ of the steel bar corrosion in the concrete structure and the chloride concentration $C$ at the time of the steel bar surface, the limit state function of the corrosion of the concrete structure can be written as follows:

$$
G=C_{c r}-C
$$

The time-dependent probability of failure for concrete structures in marine environments concerning the durability limit state can be expressed as:

$$
P_{f}=P(G<0)=P\left(C_{c r}-C<0\right)
$$

According to Eq. (5), the time-dependent reliability index of concrete structures in marine environments concerning the durability limit state can be expressed as:

$$
\beta=\Phi^{-1}\left(1-P_{f}\right)
$$


The First Order Reliability Method (FORM) addresses the reliability problem defined in Eqs. (5) and (6) by means of two key operations: one is to find the design point in the uncorrelated standard normal space; the other is to approximate the limit state surface at this point and make use of the properties of the standard normal space to obtain the probability estimate. The reliability index $\beta$ is obtained by linearizing the performance function at the point on the limit state surface nearest the origin, which can be described as the following constrained optimization problem:

$$
\left\{\begin{array}{l}
\min \beta=\sqrt{\left\{\xi^{*}\right\}^{\mathrm{T}}\left\{\xi^{*}\right\}} \\
G\left(\left\{\xi^{*}\right\}\right)=0
\end{array}\right.
$$

Where, $\left\{\xi^{*}\right\}$ is the design point vector in the uncorrelated standard normal space, which can be determined by:

$$
\{\xi\}_{k+1}=\left[\{\xi\}_{k}^{\mathrm{T}}\{\alpha\}+b\right]\{\alpha\}
$$

Where, $\{\xi\}_{k+1}$ and $\{\xi\}_{k}$ are dnotes the design points for two adjacent iteration steps. $b$ and $\{\alpha\}$ dnotes respectively the step size and the search direction vector defined by:

$$
\begin{gathered}
b=\frac{G\left(\{\xi\}_{k}\right)}{\left\|\nabla G\left(\{\xi\}_{k}\right)\right\|} \\
\{\alpha\}=-\frac{\nabla G\left(\{\xi\}_{k}\right)}{\left\|\nabla G\left(\{\xi\}_{k}\right)\right\|}
\end{gathered}
$$

Where, $\nabla G\left(\{\xi\}_{k}\right)$ is the gradient vector of the limit state surface in the standard normal space.

The iteration will be stopped when $\left|\beta_{k+1}-\beta_{k}\right| / \beta_{k} \leq 10^{-3}$ is satisfied, and $\beta_{k+1}$ will be considered as the convergence value of the reliability index.

In order to reflect the importance of the random variables to the reliability index of concrete structures in marine environments concerning the durability limit state, a importance factor can be defined by:

$$
I_{i}=\alpha_{i}^{2}
$$

Since, $\sum \alpha_{i}^{2}=1$, the importance factor is expressed as a percentage value. The FORM importance factors offer a way to rank the importance of the input variables with respect to the probability of corrosion.

\section{Numerical example}

A rectangular concrete column is located in a splash zone with a section size of $b \times h=500 \mathrm{~mm} \times 500$ $\mathrm{mm}$ and a concrete cover depth of $60 \mathrm{~mm}$. According to the "Guide to Durability Design and Construction of Reinfoced Structures" of China, the surface concentration $C_{\mathrm{s}}=0.8 \%$, and the threshold concentration $C_{\mathrm{cr}}=0.05 \%$. Assuming that the initial chloride concentration in concrete $C_{0}=0$, the chloride diffusion coefficient of concrete $D_{0}=1.0 \times 10^{-12} \mathrm{~m}^{2} / \mathrm{s}=31.536 \mathrm{~mm}^{2} /$ year, and the age factor $n=0.3$. The statistical characteristics of random parameters are shown in Table 1 . This example will verify the feasibility and accuracy of the proposed time-dependent reliability method for concrete structures.

According to the compensation length theory proposed in[15], the two-dimensional chloride diffusion control region can be divided for the cross-section of the rectangular column as shown in Fig. 1 (the hatched area indicated by a small square in Fig. 1 , and the rebar in the domain is represented by S2. ), located at the four corners of the column, and one-dimensional diffusion control 
region of chloride (the hatched area shown by fine dots in Fig. 1, S1 in the field) is located away from the column corner region.

The service life of S1 and S2 can be calculated using the time-dependent reliability model based on the one-dimensional and two-dimensional chloride diffusion models.

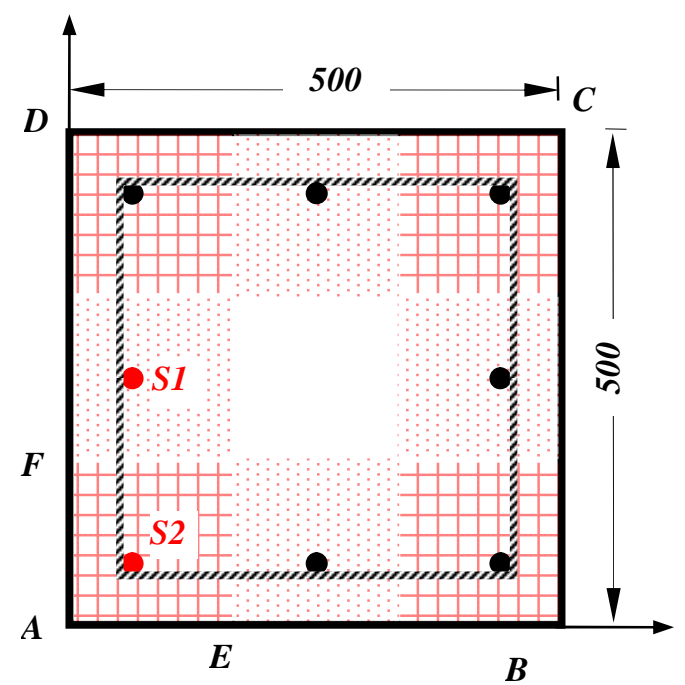

Fig.1 Control areas of concrete column

Table 1 Statistical characteristics of random variables

\begin{tabular}{lccc}
\hline Parameters & Mean & Standard deviation & Distribution type \\
\hline$D / \mathrm{mm}^{2} /$ year & 31.536 & 0.2 & Normal \\
$d / \mathrm{mm}$ & 60 & 0.2 & Normal \\
$C_{\mathrm{s}} / \%$ & 0.8 & 0.3 & Normal \\
$C_{\mathrm{cr}} / \%$ & 0.05 & 0.2 & Normal \\
$n$ & 0.3 & 0.2 & Normal \\
\hline
\end{tabular}

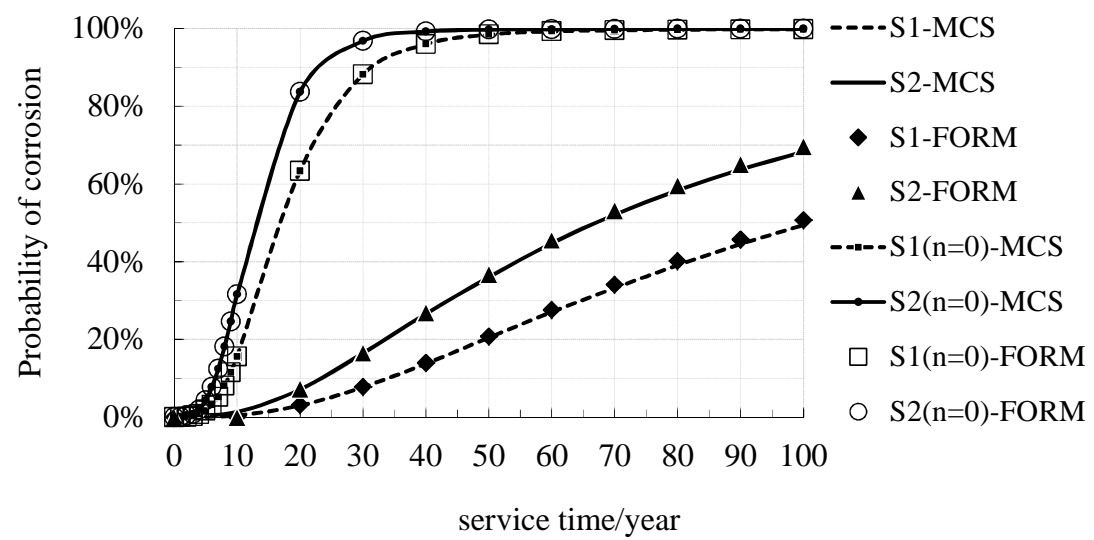

Fig.2 Corrosion Probability of Reinforced Steel in Two Diffusion Control Areas

Fig. 2 shows the corrosion probabilities of S1 and S2 at different service time. The "MCS" represents the Monte Carlo sampling method. The number of samplings in this paper is 100,000. The "FORM" represents the time-varying reliability method established in this paper based on a First-Order Reliability Method. " $n=0 "$ means that the age factor of the diffusion coefficient is ignored. It can be seen from the figure that the results of the reliability analysis method established in this paper are in good agreement with the MCS results, which proves the feasibility and accuracy of the proposed method. 
Fig. 2 also shows the influence of the age factor to the service life of S1 and S2. If $10 \%$ is taken as the target failure probability of the structure (corresponding reliability index $\beta=1.3$ ), the service life corresponding to $\mathrm{S} 1$ and $\mathrm{S} 2$ are 8.5 years and 6.4 years, respectively, regardless of the age factor of the diffusion coefficient. The values of the age-dependent attenuation coefficient of the value of diffusion coefficient and its variability are calculated to be 34 years and 23.3 years respectively for the service life of S1 and S2, which are increased by $300 \%$ and $266 \%$ respectively. Ignoring the attenuation of the diffusion coefficient, the service life of concrete structures will be seriously underestimate. Therefore, the two-dimensional diffusion model, as well as the age factor of diffusion coefficient, must be used in the durability anslysis of the concrete columns.
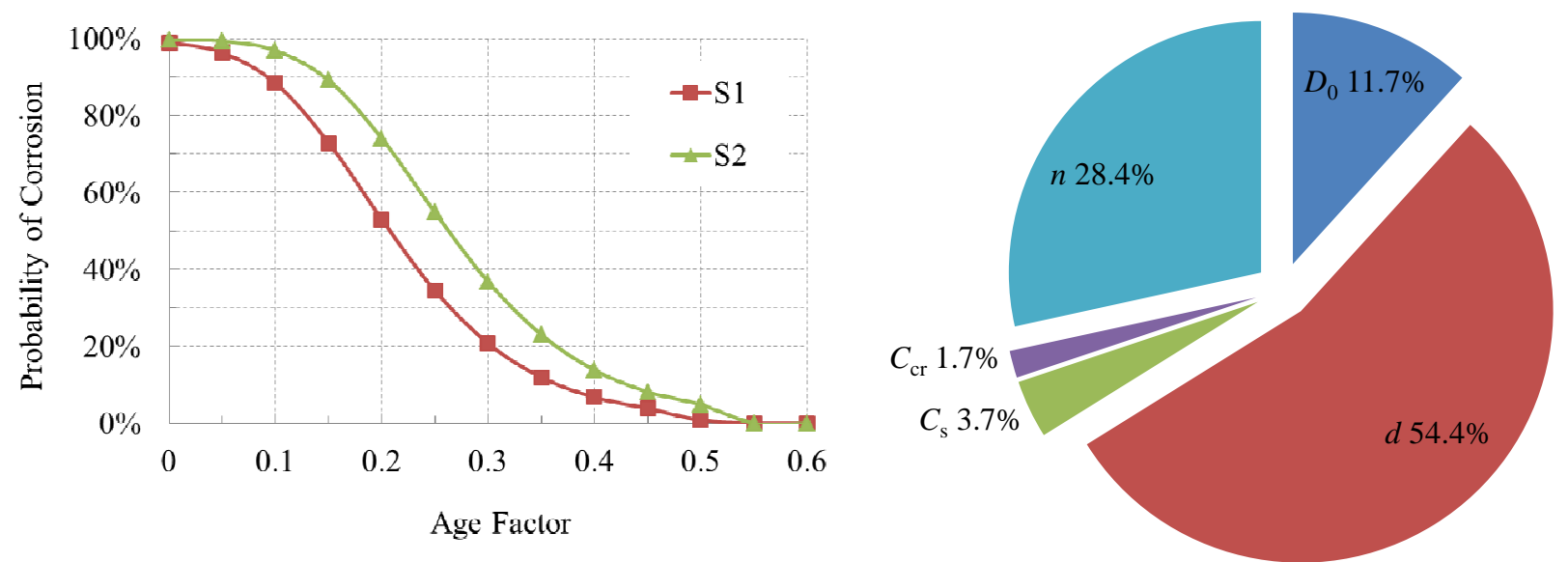

Fig.3 Corrosion probability for different age factors Fig.4 Importance factors of random parameters

The corrosion probability of S1 and S2 with different age factors at 50 years is calculated and the results are shown in Fig.3. It can be seen from the figure that with the increase of the age factor, the corrosion probability of $\mathrm{S} 2$ is always greater than that of $\mathrm{S} 1$. If the designed service life of the concrete structure is 50 years, the age factor of $\mathrm{S} 1$ cannot be less than 0.36 , but $\mathrm{S} 2$ no less than 0.44 . Therefor, the two-dimensional diffusion control regions requires more faster attenuation of the concrete than one-dimensional diffusion control regions.

Taking the concrete collomn with service time of 50 years as an example. The importance factor of reliability index with respect to the random variables are shown in Fig. 4. Through the importance factor, the influence of various parameters can be clearly sorted. It is obvious that the cover depth $d$, the age factor $n$, and the diffusion coefficient $D_{0}$ have significant influence on the reliability.

\section{Conclusions}

This paper introduces the age factor of chloride diffusion coefficient of concrete, and simultaneously considers the randomness of the chloride diffusion coefficient, cover depth, surface concentration, threshold concentration, and age factor. Based on the FORM, a time-dependent reliability analysis model for concrete structures under chloride environment is established. The results show that the time-dependent reliability model established in this paper is in good agreement with the Monte Carlo sampling method. The results of example show that the corner region of the concrete structure must be designed and calculated based on the two-dimensional diffusion model, and the attenuation characteristics and randomness must be considered for the concrete structures in chloride environment. It is obvious that the cover depth, the age factor, and the diffusion coefficient have significant influence on the reliability.

\section{Acknowledgements}

This work was financially supported by the Qinzhou University Scientific Research Foundation for Advanced Talents (2017KYQD121). 


\section{References}

[1] Clifton JR. ACI Materials Journal. Vol. 99(1993), p.661.

[2] Thoft CP. Engineering structures. Vol.20 (1998), p. 1004.

[3] SU Q, WAN XM, ZHAO TJ. Concrete. (2010), p, 16. (in chinese)

[4] Saassouh B, Lounis Z. Cement \& Concrete Composites. Vol. 34(2012), p.1082.

[5] Song H, Pack S, Ann KY. Construction and Building Materials. Vol. 23(2009), p.3270.

[6] Liu RG, Chen Y ,Cao DF. Journal of Jiangsu University. Vol. 30(2009), p.86. (in chinese)

[7] Zhang W, Dong ZL, Lu H. Port and Waterway Engineering. (2009), p. 35. (in chinese)

[8] Yunsheng Z. Journal of Wuhan University of Technology. Vol. 26(2011), p.978.

[9] Xiao JZ, Ying JW. Journal of Tongji University. (2012), p.1051. (in chinese)

[10] Yan YD, Jin WL, Wang HD. Journal of Zhejiang University. Vol. 45(2011), p. 2127. (in chinese)

[11]Zhang SF, Lu , CH, Chen Y. Engineering Mechanics. Vol.29 (2012), p. 97.

[12] Guo F, Sun W, Chen SD. Science and Technology of Overseas Building Materials. Vol. 28(2007), p.29. (in chinese)

[13] Yang LF, Chen Z, Wang Y. Journal of The Chinese Ceramic Society. Vol. 37(2009), p.1110. (in chinese)

[14] Frier C, Sorensen JD. Applications of Statisics and Probability In Civil Engineering. (2007), p. 135.

[15] Yang LF, Chen Z, Gao Q, et al. Acta Mechanica. Vol. 224(2013), p.123.

[16] Yu B, Ning CL, Li B. China Ocean Engineering. Vol. 31(2017), p.63. 